\title{
REPRESENTING DYNAMIC SOCIAL NETWORKS IN DISCRETE EVENT SOCIAL SIMULATION
}

\author{
Jonathan K. Alt \\ Stephen Lieberman \\ Modeling, Virtual Environments and Simulation (MOVES) Institute \\ Naval Postgraduate School \\ Monterey, California 93943, USA
}

\begin{abstract}
One of the key structural components of social systems is the social network. The representation of this network structure is key to providing a valid representation of the society under study. The social science concept of homophily provides a conceptual model of how social networks are formed and evolve over time. Previous work described the results of social simulation using a static homophily network. In order to gain the full benefit of modeling societies a representation of how the social network changes over time is required. This paper introduces the implementation of a dynamic homophily network, along with a case study exploring the sensitivity of model outputs to the parameters describing the network and applying social network change detection methods (SNCD) to model output.
\end{abstract}

\section{INTRODUCTION}

Understanding the motivations that cause individuals to form ties with other members of society has been a topic in the social science literature for some time (National Research Council 2008). The potential benefits to society of an understanding of the foundations of social structure by decision makers are numerous, from the development of strategies to combat gang violence to understanding how messages promoting the value of education might best reach at risk youth (Epstein 2006). This understanding of social networks and how they form and change over time is also essential to success in stability and counterinsurgency operations, where the center of gravity rests not with some military objective, but the civilian population itself (Department of the Army 2006). The social science principle of homophily provides a conceptual model grounded in years of empirical data to use for this purpose (S. Lieberman \& J. Alt 2010).

Insight into a society using homophily as a guiding principle can be achieved at points in time through the use of polling and survey data, but the most interesting questions typically lie in the space in between these snapshots in time (S. Lieberman \& J. Alt 2010). Changes in social network structure can be measured using homophily, but homophily does not provide good insight into the causal mechanisms behind these changes. Social simulations grounded on solid conceptual models from the social sciences, such as homophily, can assist the social scientist, and the public policy or military decision maker, in gaining a better understanding of the complex adaptive system that is society. Discrete event social simulations provide a fully traceable implementation of these concepts that readily accommodates the varying timescales at which events unfold within society (Jonathan Alt \& Stephen Lieberman 2010b). Other alternatives, such as systems dynamics could be used to examine the issues explored here at a macro level of analysis, but the DES agent based approach was chosen due to its ability to scale up or down in resolution. 


\section{Alt and Lieberman}

Previous work has explored methods for generating social structure from survey data and described its static implementation within discrete event social simulation (Jonathan Alt et al. 2010). This served as a logical starting point in preparation for the implementation of a dynamic social network, a network whose topology changes over the course of a scenario run, based the implementation of a sound conceptual model from the social sciences. This paper will provide an overview of a conceptual model for social simulation and its implementation in discrete event social simulation as background, propose the modifications to the conceptual model and describe the implementation of a dynamic social network, and provide a case study to explore the implementation's sensitivity to variations in the modeling parameters and characterize its performance using social network change detection (SNCD).

\section{CONCEPTUAL MODEL}

In order to provide the reader context for the current work, a brief discussion of the conceptual model of society and its implementation within discrete event social simulation is provided. A brief discussion of the data required to populate the homophily network is also included. A full discussion of methods to develop social structure from various data sources and the development of social simulations scenarios in general can be found in separate published work by the authors (Jonathan Alt \& Stephen Lieberman 2010a; Jonathan Alt \& Stephen Lieberman 2010c; Jonathan Alt et al. 2009).

The conceptual model typically refers to the causal relationships of a phenomena from the physical world derived from empirical data. In this social science setting the conceptual model bears the same connotation, with the difference lying in the level of acceptance of the theoretical basis of the conceptual model components (Alt et al. 2010). Since one of the frequent critiques of social simulations is the validity of the conceptual model, some discussion is devoted to this topic. Note that one of the unique appeals to the social science community of social simulations is the potential for the use of these tools as venues for experimentation to gain a better understanding of the interaction between the conceptual models of disparate social phenomena. The opportunity to understand how empirically developed theories accounting for the subcomponents of a society interact at the societal level is not often available in a real world setting. One of the key components of any conceptual model of society is necessarily related to the rules governing the interactions between agents, in other words the rules governing the social network (Jonathan Alt \& Stephen Lieberman n.d.).

The conceptual model of society necessarily leverages social science theory to provide an empirically grounded construct for use as a foundation for developing an abstraction of society. This conceptual model, an abstract representation of society, then serves as the guide for the representation of society at the appropriate level of fidelity for the particular use case. Multiple implementations at varying levels of fidelity and flexibility might then be built from the same conceptual model ( $\mathrm{J}$ Alt et al. 2010). Given the multiple, sometimes competing theories that exist in the social science community to explain the phenomena, it is important to use generally accepted social theories backed by empirical evidence from the social science literature when possible.

The conceptual model used in this case was introduced previously by the authors, but it is worth a short review to show the reader the central role that social structure plays in any implementation of a societal model (J Alt et al. 2010; Jonathan Alt \& Stephen Lieberman n.d.). The most basic elements of society that any social simulation must represent are the population itself, the economy, the government, the infrastructure and some notion of the influence of external societies, dependent on the scope (see figure 1). If the society is viewed as a closed system then the external influences of other societies might appropriately excluded. If the influence of outside groups is important then including some notion of this influence within the ecosystem that is society is appropriate (Mansoor 2007; Kilcullen 2006). 


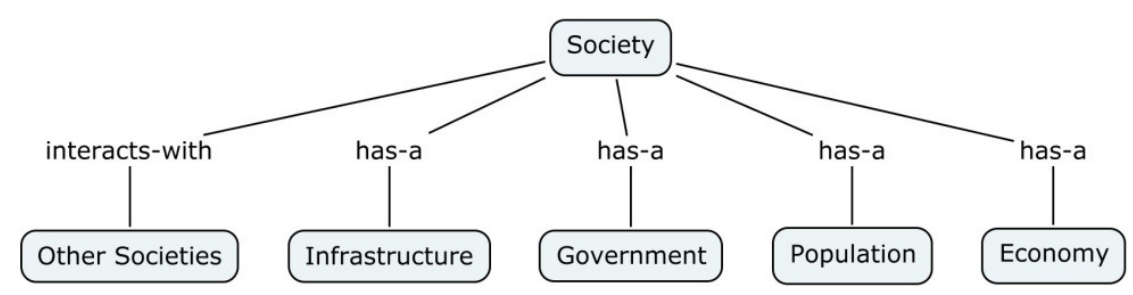

Figure 1: Conceptual model of society.

The population can be viewed as individuals and groups of individuals. The individuals themselves must have a conceptual model of their internal state and the society must provide rules for the interaction between individuals. The conceptual model of the internal state must account for how the individual interprets information from the world and how the individual selects behaviors that manifest themselves in the world and have the potential to impact other individuals. It is at this point in the conceptual model that the identification and justification for the use of specific theories from the social science to account for these phenomena must occur. When possible complementary conceptual model components should be used to reduce future data requirements and provide logical consistency throughout the model. The conceptual modeling diagram from above is expanded in figure 2 below, naming specific theories from the social science literature to account for the phenomena required for representation. Note that depending on the region of the world, time period under study, or available data, the modeler might choose a different set of theoretical building blocks.

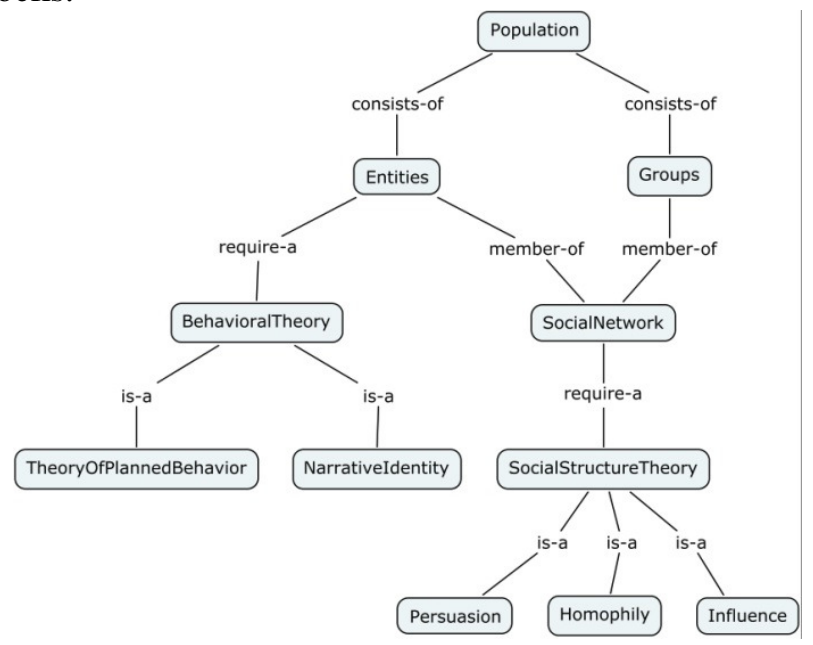

Figure 2: Expanded conceptual model of society with specified theoretical models.

The foundation of the conceptual model of internal state for each individual is the narrative paradigm. Fisher's narrative identity proposes that individual's possess unique narratives based on their experiences, socio-demographic, socio-economic and socio-cultural make-up that shape the lens through which they view the world (Fisher 1989). These narratives hold the beliefs, values and interests (BVI) that focus the lens to provide a unique perspective on events and issues within society.

The action choice component of the conceptual model is based on the theory of planned behavior (TPB) (I. Ajzen 1991). The TPB states that an individual forms the intention to take part in a behavior based on the his perception of subjective norms regarding the behavior, the individual's attitude toward the behavior, and the individual's perceived behavioral control regarding the behavior. The TPB has received extensive use in applied settings in the areas of marketing and behavior modification programs (exercise adoption, smoking cessation) (Icek Ajzen 2006). The model has an associated instrument for data collection that can be modified to accommodate additional behaviors.

The social structure component of the conceptual model is based on the principle of homophily. Homophily describes the likelihood of an interaction between two individuals in society as a function of their 


\section{Alt and Lieberman}

relevant socio-demographic, socio-economic, and socio-cultural identity (M. McPherson et al. 2001). The distance between two individuals within based on these factors defines the likelihood that they will interact. This view is complementary to the narrative identity and provides a conceptual model that is feasible to populate from empirical data. The principle itself is well grounded in with empirical studies from the social sciences. Using this concept the social structure of a society can be determined and represented as a fully connected network with the nodes representing individual members or population segments, depending on the level of abstraction, and the strength associated with the arcs describing the likelihood of an interaction between that node arc pair (Jonathan Alt et al. 2009). Depending on the needs of the modeling effort the strength of the link required for representation within the network can be specified. The next section will discuss the discrete event social simulations focusing on the social network implementation.

Discrete event simulation provides a well established mechanism for the modeling of processes of many types and has been successfully used in modeling human behavior through the use of task network models (Ahner et al. 2008). Many of the characteristics which make it useful for modeling individual human behavior, also make it an attractive candidate for use in social simulations. In particular discrete event simulation techniques facilitate the representation of social structure and the multiple interactions between agents governed by the conceptual model and the competing time scales which operate in a society. The authors discuss the benefits of discrete event simulation in this domain in greater detail in separate works (Jonathan Alt \& Stephen Lieberman 2010b; Jonathan Alt \& Stephen Lieberman n.d.). The conceptual model described above translates into the event graph representation shown below in figure 3 .

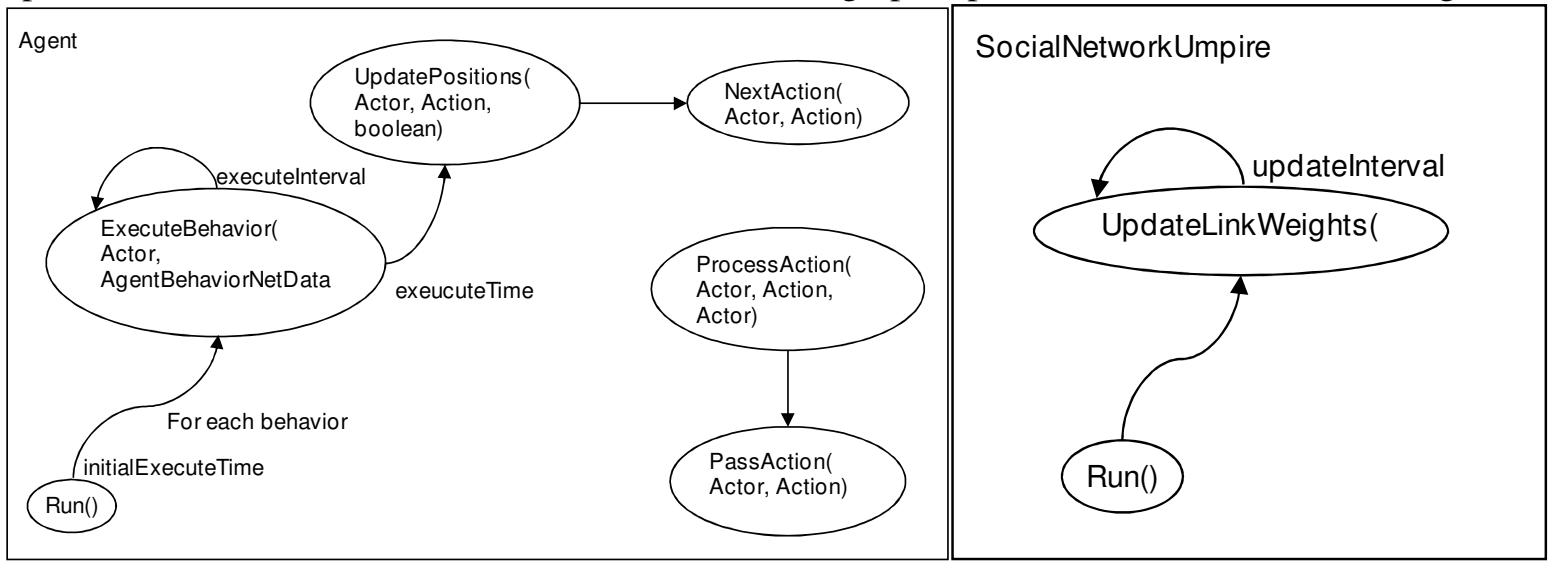

Figure 3: Event graph representation of CG model agent (left) and social network umpire (right).

The internal state model for each agent then contains a state variable for each issue represented within the social simulation. This state variable is updated the agent gains information regarding events that occur within the model through a transition function based on the BVI of the population segment that the agent represents. Similar state variables maintain the agents behavioral intention over the course of a scenario run. The link weights used to describe the social network structure are provided as parameters during scenario construction, calculating the homophily weight as a pre-processing step.

The described conceptual model and the event graph representation of it are implemented within the Cultural Geography (CG) model (Jonathan Alt et al. 2010). The CG model is a government owned, opensource, social simulation implemented in Java that leverages the Simkit capability developed at the Naval Postgraduate School (Buss 2002). The CG model is under continuous development with additional capability added based on applied analysis and research needs. The dynamic homophily implementation and subsequent case study analysis were conducted using this social simulation. 


\section{DYNAMIC SOCIAL NETWORK IMPLEMENTATION}

Social networks change over time as elements that inform the degree of likeness between two individual members of the network change (M. McPherson et al. 2001; Miller McPherson \& Ranger-Moore 1991; Miller McPherson et al. 1992; Miller McPherson et al. 2006). While some elements remain fairly static, such as most socio-demographic characteristics, other elements, such as beliefs, values and interests (BVI), are dynamic and change based on the events within the world. This component of homophily, sometimes referred to as value homophily, provides the basis for the implementation of a dynamic social network. The concept of attained attributes, such as education, also provides a model for changes in homophily over time, but is outside the scope of the current effort (Blau 1994). This section will describe the conceptual model of the dynamic component of a social network and provide a brief overview of its implementation within the discrete event framework.

\subsection{Conceptual Model}

The conceptual foundation of the social structure model remains fundamentally unchanged. Homophily persists as the theoretical basis for the determination of the likelihood of interaction between any two agents. The conceptual model is extended to include BVI which change over time, consistent with the concept of value homophily. This extension is closely aligned with the conceptual model for maintaining internal state for each individual, the narrative identity. As positions on salient issues change through internal state models the individuals degree of likeness to other individuals will be impacted. In the simple case, two otherwise homogeneous individuals who have differing views on a particularly salient issue will move further apart as their positions on that particular issue diverge. The likelihood of their interaction will decrease based on their disagreement on this issue.

\subsection{Discrete Event Implementation}

The implementation of the conceptual model of the dynamic components of homophily result in a fairly straight forward implementation (see figure 4). The implementation results in the inclusion of several state variables, issue stances from the developed scenario, in the basic homophily calculation. The implementation of this concept, however, did result in the need to calculate the all pairs link weights during runtime, rather than to simply specify the parameters of the network as scenario data. The scenario developer must also specify the update rate for the homophily network. In reality this likely occurs at different rates for each individual within a representative social network or alternatively might be triggered by significant events. In this first implementation, the update rate can be adjusted globally for the network as a parameter specified during scenario construction. The parameters, state variables and the component event graph are described below.

- Parameters:1) Socio-demographic, socio-economic, socio-cultural dimensions required to partition the population; 2) the internal issue stances, state variables to be used during the calculation of homophily; 3) weights to apply to each of these dimensions during the calculation of homophily; 3) the homophily network refresh rate.

- State variables: the link weight between each agent and all other agents within the society.

In this case each agent, $i$, possesses a vector of state variables, $I$, that describe their unique relationships with other agents, $j$, within the model. These state variables result from the calculation of the all pairs distance between agents within the model using a combination of the socio-demographic, sociocultural and socio-economic parameters, $d$, specified at the beginning of the run and the state variables describing the unique BVI related to the issues within the model, which are dynamic over the course of a simulation run. The equation used to calculate the distance between two entities, Sij, and the calculation for the resulting link weight, $W i j$, are shown below. 


$$
s_{i j}=\sqrt{\left(\frac{d_{1_{i j}}}{\max d_{1}}\right)^{2}+\left(\frac{d 2_{i j}}{\max d_{2}}\right)^{2}+\ldots+\left(\frac{d k_{i j}}{\max d_{k}}\right)^{2}+\left(\frac{I_{k_{i j}}}{\max I_{k}}\right)^{2}}
$$

This formulation of the distance calculation weights all factors equally and normalizes within each dimension. Alternatively, the dimensions could be used directly and a set of weights applied. Future research will explore the trade-offs of these approaches.

$$
w_{i j}=1-\left(\frac{s_{i j}}{\max s_{i j}}\right)
$$

\section{EXPLORATORY CASE STUDY}

In order to characterize the sensitivity of the dynamic social network to the parameters specified as starting conditions an exploratory case study was developed. This section describes the case study, the process of initializing the social structure used for this case study, the design of experiments used to explore the parameter space, and the analysis of the results.

\subsection{Case Study Description}

This work leveraged an unclassified scenario set in southern Afghanistan. In the scenario stabilizing force agents seek to influence the populations satisfaction on a number of issues while the opposing insurgent force seeks to destabilize the region by reducing the populations satisfaction on these issues. An extensive data development effort from the open source populated the model. This effort simply uses this scenario as a test case for the implementation of dynamic homophily within the CG model and due to space limitations will only be described as it relates to this implementation.

\subsection{Dynamic Value States}

The components of the homophily calculation that change over time in this case are the three issue stances tracked internally by each agent, state variables, over time. Each time the social network is updated the current value of these three state variables is used, in conjunction with the static parameters describing the agent's socio-demographic, socio-economic, and socio-cultural characteristics, to determine the current link weight between each agent in the simulation scenario. As agents receive information from observation or through the network they update their issue stances using transition functions specified at the beginning of the scenario. In this case study these transition functions take the form of Bayesian belief networks developed through subject matter expert input. The development of these networks is discussed in greater detail in other works by the authors, but in a discrete event view could be any function that describes the transition of the state variable based on input from the environment.

\subsection{Design of Experiments}

The purpose of this exploratory case study was to characterize the performance of the dynamic homophily implementation as parameters describing the starting condition changed. A nearly orthogonal Latin hypercube (NOLH) was chosen for use in this case due to its space-filling characteristics and its usefulness in identifying main effects (Sanchez \& T. W Lucas 2002; T. M Cioppa et al. 2004; Thomas Cioppa \& Thomas Lucas 2007). Since multiple parameters describe the rules for the actual implementation of the social network it is important to understand how sensitive the model results are to these starting conditions. The factors chosen for inclusion in the design included: the number of agents chosen to receive the effects of an action if no target recipient is specified (recNoTGT), the period of time during which an agent will only pass an action once to other agents in its network (doNotPassInt), the number of $\mathrm{k}$ nearest neighbors used to determine targets for message traffic $(\mathrm{k})$, the time delay between the receipt of a message and the scheduling of its passage to a recipient (comTimeClass), the time an agent will com- 


\section{Alt and Lieberman}

municate about an event (comExpire), and the interval between updates of the social network link weights (updateInt). The highest correlation between the rows and columns of this design matrix was 0.0024 . The run time of the social simulation and the proof of principle nature of the work precluded the planned replication of the design. Future work will move the simulation to a high performance computing environment more conducive to multiple replications to develop more robust statistical measures. The results provided are based on only 5 replications per design point.

Table 1: Experimental design to examine social network parameter impact on issue stances.

\begin{tabular}{|c|c|c|c|c|c|c|}
\hline Factor Name & k & comTimeClass & comExpire & recNoTGT & doNotPassint & upDateInt \\
\hline Low & 0 & 0.05 & 1 & 1 & 0 & 5 \\
\hline High & 7 & 2.5 & 14 & 30 & 7 & 25 \\
\hline \multicolumn{7}{|l|}{ Design Point } \\
\hline 0 & 2.19 & 2.5 & 11.56 & 11.88 & 1.75 & 23.75 \\
\hline 1 & 0.44 & 0.66 & $\mid 12.38$ & 17.31 & 0 & 11.25 \\
\hline 2 & 0.88 & 1.12 & 1.81 & 8.25 & 4.38 & 21.25 \\
\hline 3 & 1.31 & 1.58 & 5.06 & 30 & 3.94 & 7.5 \\
\hline 4 & 5.25 & 2.35 & 6.69 & 4.62 & 2.19 & 5 \\
\hline 5 & 7 & 0.82 & 5.88 & 24.56 & 0.44 & 20 \\
\hline 6 & 4.38 & 0.51 & 14 & 10.06 & 6.12 & 13.75 \\
\hline 7 & 3.94 & 2.19 & 10.75 & 28.19 & 5.69 & 17.5 \\
\hline 8 & 3.5 & 1.28 & 7.5 & 15.5 & 3.5 & 15 \\
\hline 9 & 4.81 & 0.05 & 3.44 & 19.12 & 5.25 & 6.25 \\
\hline 10 & 6.56 & 1.89 & 2.62 & 13.69 & 7 & 18.75 \\
\hline 11 & 6.12 & 1.43 & 13.19 & 22.75 & 2.62 & 8.75 \\
\hline 12 & 5.69 & 0.97 & 9.94 & 1 & 3.06 & 22.5 \\
\hline 13 & 1.75 & 0.2 & 8.31 & 26.38 & 4.81 & 25 \\
\hline 14 & 0 & 1.73 & 9.12 & 6.44 & 6.56 & 10 \\
\hline 15 & 2.62 & 2.04 & 1 & 20.94 & 0.88 & 16.25 \\
\hline 16 & 3.06 & 0.36 & 4.25 & 2.81 & 1.31 & 12.5 \\
\hline
\end{tabular}

\subsection{Analysis}

The purpose of the case study was to identify the main effects among the input parameters to the rules governing the social network from the design of experiments and to characterize the performance of the dynamic homophily implementation. In order to inform these the results of the designed experiment were analyzed using screening methods to identify main effects and the social network itself was examined over the course of scenario runs using standard metrics from social network analysis to characterize the changes in social network structure. The response used in the analysis of the designed experiment is the population's satisfaction on the issue of security. The aggregate response for all agents over time for each of the design points explored is shown below. Note three design points (DP) failed to produce useable results, DP 1, DP 5, and DP 14 (output files were corrupted). Following the elimination of the three failed design points, the maximum correlation between the rows and columns of the updated design matrix was 0.29 .

The analysis to identify main effects will be discussed in the next section followed by an analysis of changes over time in the social network. 


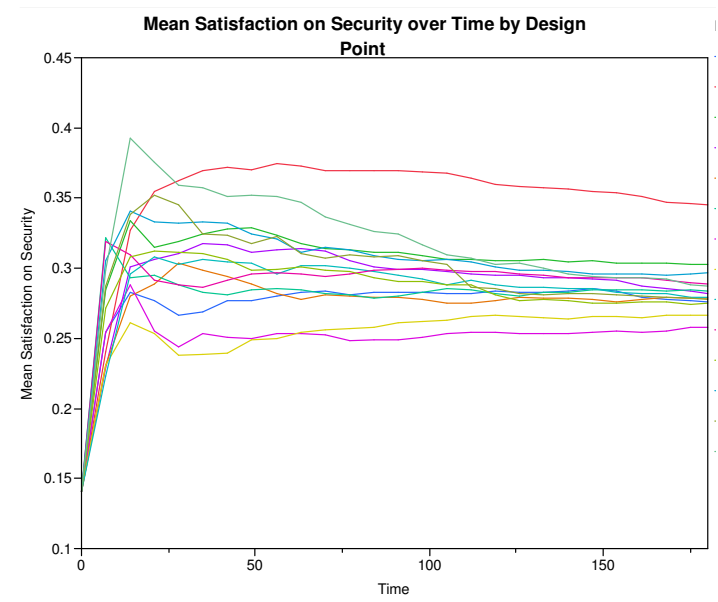

Figure 4: Mean satisfaction on security over time by design point using JMP statistical software.

\subsubsection{Identifying Main Effects}

The identification of main effects provides the simulation modeler insight into the impact of starting parameters on the measures generated over the course of the simulation run. While in some cases the impact of parameter settings are obvious, the incorporation of multiple conceptual models into a complex adaptive system can provide challenges to verifying the impact of individual model components. The exploration of these design parameters can aid the model developer in fully characterizing the use of the developed model component in as part of the larger system.

The analysis technique used in this case is stepwise regression. Stepwise regression procedures evaluate only a small number of the possible regression models by adding or deleting single regressors, or factors. The three broad categories of this technique are forward selection, backward elimination, and stepwise regression, which combines forward selection and backward elimination (Montgomery et al. n.d.). In general, forward selection begins with no terms in the model and adds a single term at a time, evaluating each term based on its partial F statistic (Montgomery et al. n.d.).

Backward elimination, in contrast, includes all possible terms and uses the partial F statistic to eliminate terms. Stepwise regression combines the techniques by proceeding with forward selection, but evaluating all terms added to the model at each step as in backward elimination. A full treatment of these techniques is provided by Montgomery (Montgomery et al. n.d.).

In order to identify the most significant factors from the designed experiment that impacted the response, the aggregate stance of the population of security at the end of the run, stepwise regression was used, examining main effects and second and third order interactions and polynomial relationships. The probability to enter the model was set at 0.15 and the probability to leave was set at 0.1 . A model resulting in an R-square of 0.99 was fit using four terms from the experimental design: $\mathrm{k}$, comExpire, recNoTGT, and doNotPassInt. The effects test results using based on the F ratio show that $\mathrm{k}$, the number of $\mathrm{k}$ nearest neighbors considered for communications, had the greatest effect of any single factor on the model outcomes. While dominated as a main effect by itself by $\mathrm{k}$, comExpire as second order interaction appears in three terms, with only $\mathrm{k}$ surpassing it with polynomial terms of second and third order. 
Table 2: Main effects test from results of stepwise regression.

\begin{tabular}{|l|r|}
\hline \multicolumn{1}{|c|}{ Term } & \multicolumn{1}{c|}{ F Ratio } \\
\hline $\mathrm{k}^{*} \mathrm{k}^{*} \mathrm{k}$ & 10292.10 \\
\hline $\mathrm{k}{ }^{*} \mathrm{k}$ & 9217.47 \\
\hline comExpire*doNotPassInt & 2712.14 \\
\hline comExpire*comExpire & 1832.94 \\
\hline comExpire*recNoTGT & 1444.78 \\
\hline recNoTGT*doNotPassInt & 1332.45 \\
\hline $\mathrm{k}$ & 759.51 \\
\hline recNoTGT*recNoTGT & 230.42 \\
\hline comExpire & 28.31 \\
\hline doNotPassInt & 21.03 \\
\hline recNoTGT & 18.19 \\
\hline comExpire*recNoTGT*doNotPassInt & 2.17 \\
\hline
\end{tabular}

As the period of time increases during which an event will be discussed, comExpire, the impact of the period of time over which only a single message will be passed about any single event, doNotPassInt, increases. The same relationship exists between comExpire and the number of individual agents that receive generic message traffic, recNoTGT.

The results of the analysis for main effects show that the number of $\mathrm{k}$ nearest neighbors used to determine the number of message recipients for agent to agent communications was the dominant factor in the experimental design. The number of recipients of non-targeted message traffic showed significance as an interaction term as did the period of time that agents would schedule communications about an event following its occurrence. Given that these terms impact the volume of communications events, which directly affect the agent's update mechanism for internal state, their significance is expected. The update rate for the link weights did not appear as a significant term in the model. The likely cause of this is the relative stability of the aggregate security stance within the model following the 25th time period. Relatively small changes occur within issue stances following each event, making it unlikely that significant changes would occur to the calculated link weights given the current methods for updating issue stances. In order to further explore the implementation of the dynamic social network, methods for social network change detection are explored further below for a single model run.

\subsubsection{Social Network Change Detection}

The detection of change dynamic social networks has received recent attention in the literature. In particular, work by McCulloh and Carley focuses on change detection in dynamic social networks (McCulloh 2009; McCulloh \& Carley 2008a; McCulloh \& Carley 2008b). McCulloh proposes and demonstrates the use of techniques from statistical process control, such as control chart methods, as a means of detecting change in dynamic social networks (McCulloh 2009). In this case study we apply his social network change detection (SNCD) methodology to the simulation output of the dynamic social network implemented within discrete event simulation using output data from design point number 16 . In this proof of principle work, the methodology is applied to the output from only a single design point due to space and time constraints.

Data describing the social network (link weights greater than 0.5) was captured at a set interval, every 7 time periods, over the course of the model run, resulting in 27 unique networks, but with consistent nodes. Using this data we can calculate measures describing the structure of the network and examine how these measures change over time. Treating these as process outputs control chart techniques are then applied to identify those points in time when the network structure has changed as measured by the network statistics. Multiple control chart techniques exist, but McCulloh shows that the cumulative sum (CUSUM) control chart is effective in detecting small changes in networks (McCulloh \& Carley 2008b). The network measures chosen for the application of SNCD can vary based on the needs of the analysis, but in this case the measures used by McCulloh are mirrored here. The measures used are the average, 


\section{Alt and Lieberman}

maximum, and standard deviation of the network betweenness and closeness. Each of these metrics is discussed briefly here, with a full discussion provided by McCulloh (McCulloh 2009).

Betweenness centrality, $b_{k}$, provides a measure of how often a node, $k$, falls on the shortest path between two other nodes, $i$ and $j$ in the network. The number of paths between $i$ and $j$ that also cross $k, g_{i j k}$, is divided by the total number of paths from $i$ to $j, g_{i j}$. In practical application nodes with a high betweenness serve as logical dividing points to create sub-graphs. The betweenness calculation for a single node, $k$, is shown below. The average betweenness centrality over all nodes in the network for each time period is used as a measure of network change in this case.

$$
b_{k}=\sum_{i, j} \frac{g_{i k j}}{g_{i j}}
$$

Closeness measures the sum of the distance, g, from a single node, $k$, to all other nodes, $i$, in the network. Nodes with a high closeness centrality are well positioned to influence the rapid dissemination of information. The closeness centrality calculation for a single node is shown below. As before the average closeness centrality over all nodes in the network for each time period is used to measure change.

$$
c_{k}=\frac{\min _{k}\left\{\sum_{i=1}^{n} g_{k i}\right\}}{\sum_{i=1}^{n} g_{k i}}
$$

The results of the application of the SNDC methodology the output of the dynamic social network is shown below. Space restrictions prevent a full discussion of the CUSUM control chart methodology, however, it is important to note that techniques such as SNDC are required to both verify model components from social simulation and to gain a full understanding of model results in order to better inform decision makers. For comparison a control chart using Xbar is shown at left and the CUSUM at right. Note the out of control behavior in the CUSUM chart for betweenness.
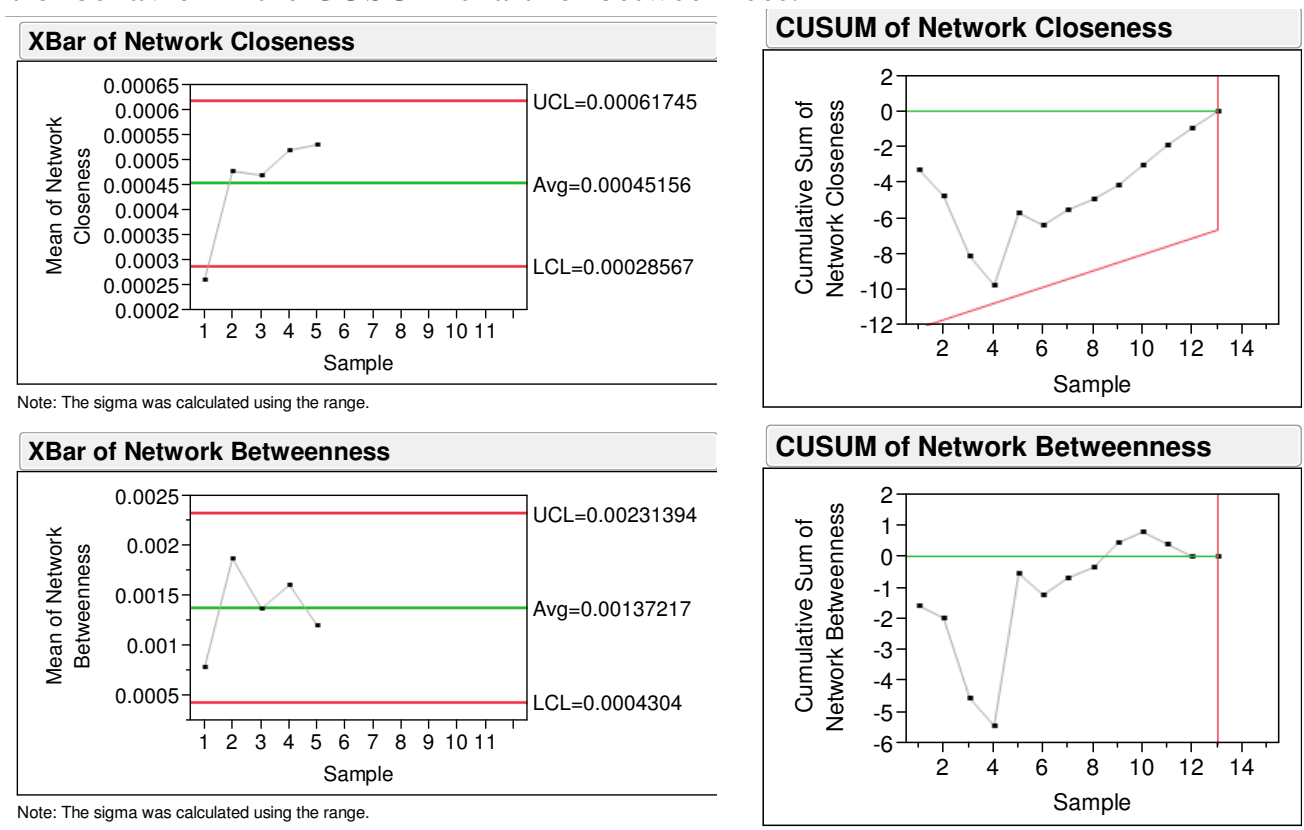

Figure 5: XBar control chart (left) and CUSUM (right) of network measures over time.

\section{CONCLUSIONS}

This paper described a conceptual model for a dynamic social network and its implementation within discrete event social simulation. The implementation of a dynamic social network within social simulations based on the conceptual model provided by value homophily and described above provides the ca- 
pability to explore how the relationships between individuals and population groups within a society change over time and in response to a variety of initial conditions. The use of social simulations in support of public policy decision makers requires continued efforts on the part of the modeling and simulation community to ensure that models provide capabilities capable of providing insights, while ensuring they are based on sound empirically derived conceptual models. This paper provides a contribution to the simulation body of knowledge by describing the implementation of a dynamic social network based on homophily and the application of SNCD methods to the output of discrete event social simulations. Future efforts will seek develop scenario's using longitudinal data from survey efforts in order to compare model outputs to empirical data and to extend SNCD methods by exploring the application of these techniques to empirically derived homophily networks.

\section{REFERENCES}

Ahner, D.K. et al. 2008. Incorporating information networks into military simulations. In Proceedings of the 40th Conference on Winter Simulation. pp. 133-144.

Ajzen, I. 1991. The theory of planned behavior. Organizational behavior and human decision processes, 50(2), 179-211.

Ajzen, I. 2006. Constructing a TpB Questionnaire: Conceptual and Methodological Considerations.

Alt, J., S. Lieberman, and C. Blais. 2010. A Use-Case Approach to the Validation of Social Modeling and Simulation. In Agent Directed Simulation Symposium, Spring Simulation Multiconference. 2010 Spring Simulation Multiconference. Orlando, FL: Society for Modeling and Simulation International.

Alt, J. et al. 2010. The Cultural Geography Model: Evaluating the Impact of Tactical Operational Outcomes on a Civilian Population in an Irregular Warfare Environment. Journal of Defense Modeling and Simulation, (Special Issue on Counter-Insurgency).

Alt, J., L. Jackson, and S. Lieberman. 2009. The Cultural Geography Model: An Agent Based Modeling Framework for Analysis of the Impact of Culture in Irregular Warfare. In International Conference on Computational Cultural Dynamics. Third International Conference on Computational Cultural Dynamics. Maryland.

Alt, J. and S. Lieberman. 2010. Agent Frameworks for Discrete Event Social Simulations. In Behavior Representation in Modeling \& Simulation. Behavior Representation in Modeling \& Simulation 2010.

Alt, J. and S. Lieberman. 2010a. Developing Cognitive Models for Social Simulation from Survey Data. In Social Computing, Behavioral Modeling and Prediction. 2010 International Conference on Social Computing, Behavioral Modeling, and Prediction. Maryland: Springer.

Alt, J. and S. Lieberman. 2010b. Exploring the Implications of Time in Discrete Event Social Simulations. Association for the Advancement of Artificial Intelligence Spring Symposium 2010.

Alt, J. and S. Lieberman. 2010c. Modeling the Theory of Planned Behavior from Survey Data for Action Choice in Social Simulations. In Behavior Representation in Modeling \& Simulation. Behavior Representation in Modeling \& Simulation 2010. Charleston, SC.

Blau, P. 1994. Structural Context of Opportunities, University of Chicago Press.

Buss, A. 2002. Simkit: component based simulation modeling with Simkit. In Proceedings of the 34th conference on Winter simulation: exploring new frontiers. pp. 243-249.

Cioppa, T.M., T.W. Lucas, and S.M. Sanchez. 2004. Military applications of agent-based simu- 
lations. In Proceedings of the 36th conference on Winter simulation. p. 180.

Cioppa, T. and T. Lucas. 2007. Efficient Nearly Orthogonal and Space-Filling Latin Hypercubes. Technometrics, 49(1), 45.

Department of the Army, 2006. Counterinsurgency, Epstein, J. 2006. Generative Social Science, Princeton, NJ: Princeton University Press. Fisher, W. 1989. Clarifying the Narrative Paradigm. Communication Monographs, 56(1), 55-58. Kilcullen, D. 2006. Counter-insurgency Redux. Survival, 48(4), 111-130.

Lieberman, S. and J. Alt. 2010. Developing Social Networks for Artificial Societies from Survey Data. In Social Computing, Behavioral Modeling and Prediction. Springer.

Mansoor, P. 2007. A New Counterinsurgency Center of Gravity Analysis: Linking Doctrine to Action. Professional Military Review, Journal of the U.S. Army.

McCulloh, I. 2009. Detecting Changes in a Dynamic Social Network. Carnegie Mellon University.

McCulloh, I., and K.M. Carley. 2008a. Dynamic Network Change Detection, West Point, NY: United States Military Academy.

McCulloh, I.A. and K.M. Carley. 2008b. Social network change detection. Center for the Computational Analysis of.

McPherson, M., L. Smith-Lovin, and J.M. Cook. 2001. Birds of a feather: Homophily in social networks. Annual review of sociology, 27(1), 415-444.

McPherson, M., P. Popielarz, and S. Drobnic. 1992. Social Networks and Organizational Dynamics. American Sociological Review, 57.

McPherson, M. and J. Ranger-Moore. 1991. Evolution on a Dancing Landscape: Organizations and Networks in Dynamic Blau Space. Social Forces, 70(1), 19-42.

McPherson, M., L.Smith-Lovin, and M. Brachears. 2006. Social isolation in America: Changes in core discussion networks over two decades. American Sociological Review, 71(3), 353-375.

Montgomery, D., E. Peck, and G. Vining. Introduction to Linear Regression Analysis 4th ed., New Jersey: John Wiley and Sons.

National Research Council, 2008. Behavioral Modeling and Simulation: From Individuals to Societies, National Academies Press.

Sanchez, S.M., and T.W. Lucas. 2002. Exploring the world of agent-based simulations: simple models, complex analyses: exploring the world of agent-based simulations: simple models, complex analyses. In Proceedings of the 34th conference on Winter simulation: exploring new frontiers. pp. 116-126.

\section{AUTHOR BIOGRAPHIES}

JONATHAN ALT is a student at the Modeling, Virtual Environments, and Simulation Institute at the Naval Postgraduate School. His research interests include cognitive modeling and the military application of agent based social simulations.

STEPHEN LIEBERMAN is researcher at the Modeling, Virtual Environment, and Simulation Institute at the Naval Postgraduate School. His research interests include social network analysis, social modeling, and artificial societies. 